\title{
Preface: ICNIRS 2017 proceedings
}

\section{Søren Balling Engelsen, Klavs Martin Sørensen and Frans van den Berg}

www.icnirs2017.com

The ICNIRS conference series have proven to be very sustainable because of many factors, but most of all because NIR spectroscopy has proven to be an extremely versatile, robust and informative sensor technology. This in turn creates lots of attention from researchers from practically all fields of science and perhaps even more pronounced, from all corners of advanced technology providers. The ugly duckling ${ }^{1}$ amongst spectroscopies has indeed turned into a swan and revolutionised quality control in practically all areas of food, agricultural and pharmaceutical production. The latest fruit on the tree is the integration of a NIR spectrometer with the omnipresent mobile phones. These NIR sensors are not yet at an adequate performance level for more than very simple applications, but they will definitively develop rapidly and only the future can predict what will happen when NIR gets democratised and we get powerful NIR sensors in our daily life. Last but not least, the ICNIRS conference would not survive without the dedicated interest of the instrument and supplier industries. A total of 502 persons from all over the world attended the ICNIRS2017 meeting held in the Bella Center venue in Copenhagen. The sponsor interest and support were better than ever with 2 platinum and 14 gold sponsors. We owe a particular thank to the two platinum sponsors BRUKER and FOSS, who made the ICNIRS a lively conference economically, technically and socially.

It is difficult to point out highlights from the conference, but it is worth mentioning the three ICNIRS awards which were given during the meeting. The former president Pierre Dardenne from the Walloon Agricultural Research Center in Belgium was awarded the Karl Norris Award for his "lifetime achievements" in the NIR spectroscopy field. Professor Satoru Tsuchikawa from Nagoya University in Japan was awarded the 2017 Tomas Hirschfeld award for outstanding achievements in the application of NIR spectroscopy to woods and Professor Yukihiro Ozaki from Kwansei-Gakuin

\section{Correspondence}

S.B. Engelsen (se@food.ku.dk)

doi: $10.1255 /$ nir2017.001

Citation: S.B. Engelsen, K.M. Sørensen and F. van den Berg, "Preface: ICNIRS

2017 proceedings", in Proc. $18^{\text {th }}$ Int. Conf. Near Infrared Spectrosc., Ed by

S.B. Engelsen, K.M. Sørensen and F. van den Berg. IM Publications Open,

Chichester, pp. 1-2 (2019). https://doi.org/10.1255/nir2017.001
University in Japan was awarded the ICNIRS fellowship. The theme of the conference was NIR spectroscopy at work in industry, and we had divided the scientific programme into eight topics: Dairy and Food, Pharma and Biotech, Theory and Instrumentation, Water, Soil and Environment, Process Analytical Technology, Agriculture, Hyperspectral Imaging and Chemometrics. For each topic, one plenary lecture and from three to eight oral presentations were selected, and in addition to this, around 200 posters were presented at the conference. The selected plenary lecturers were: Steve Holroyd from New Zealand who gave an overview of the use of NIRS in the dairy industry, Erik Skibsted from Denmark who talked about the growing opportunities for using NIRS in the pharma industry with emphasis on new developments in personalised medicine, Frans van den Berg who talked about process control using NIRS, Christian Huck from Austria who talked about Theoretical and Technical Advancements of NIRS including the loss of information when going to miniaturised instruments, Véronique Bellon-Maurel from France who talked about the possible use of NIRS in the future digital agriculture, Wouter Saeys from Belgium who talked about the use of NIRS in precision agriculture and vertical farming, Maria Angela Franceschini, from Harvard Medical School in US, who talked about the use of NIRS (aka diffuse correlation spectroscopy) in clinical neuroscience and Harald Martens from Norway who talked about the use of big data and chemometrics to NIRS data. A special Danish honour session was made for Professor Lars Munck who has pioneered the use of NIR spectroscopy; first at The Carlsberg Laboratories and later, in 1990, he introduced it in the academic settings of the Royal Veterinarian and Agricultural University. Lars Munck gave a lecture with the title: "The Unreasonable Efficiency of Near Infrared Spectroscopy in Biology" which can be seen at the ICNIRS 2017 homepage (www.icnirs2017.com).

\section{(C) 2019 The Authors}

This licence permits you to use, share, copy and redistribute the paper in any medium or any format provided that a full citation to the original paper is given.

ISBN: 978-1-906715-27-4 
All in all, the conclusion on the ICNIRS conference is that NIRS continues to have a "shining" future and we can look forward to lots of new trends in the application of NIRS in the near and far future. It is clear that Pharma and Chemical Engineering are leading the economy of NIRS developments, but soon the drag for sustainable production and circular economy will require more frequent measurements of practically all production streams including ingredients, water, waste and products. As a versatile, robust and informative sensor technology such as NIRS should have an important role to play here. As already mentioned, miniaturisation of NIRS has the potential to become an omnipresent sensor technology in our smartphones, in monitoring processes and in drones. The opportunities are immense and the instruments are improving and coming down in cost all the time-it will become a serious gamechanger when the quality of the NIR spectra improves. Having NIR sensors everywhere also requires that the gigantic data streams can be analysed and converted to information and it will be most interesting to observe the merging of new devel- opments in deep learning and machine learning with NIRS. We have already started to observe development towards automated calibrations using local modelling and the cloud.

Unfortunately, the present ICNIRS proceedings cannot reflect all the interesting topics from the conference, but we hope that it can give some status on the current state of art in the NIRS community. The selected papers have been mildly peer reviewed by us, but we have decided to leave the manuscripts as intact as possible in order to give the reader a more direct report from the authors of the manuscripts. We hope that you will find the ICNIRS2017 proceeding helpful and enjoy reading it.

Looking forward to seeing you all at the ICNIRS conference on the Gold Coast in Australia in September 2019, warmest regards.

\section{References}

1. H.C. Andersson, The Ugly Duckling (1844). 